\title{
Evaluación de plantaciones de Tabebuia rosea (Bertol.) DC. y Swietenia macrophylla King en el Centro- Occidente de México
}

\author{
Assessment of Tabebuia rosea (Bertol.) DC. and \\ Swietenia macrophylla King plantations in Central- \\ Western Mexico
}

\author{
Agustín Rueda Sánchez ${ }^{1}$, Juan de Dios Benavides Solorio ${ }^{1}$, J. Trinidad Sáenz Reyes ${ }^{2}$, \\ H. Jesús Muñoz Flores², David Castillo Quiroz ${ }^{3}$ y Jesús Eduardo Sáenz Ceja**
}

\begin{abstract}
The success in the establishment and development of forest plantations depends on the fact that species are appropriate for the agroclimatic conditions of plantation sites. This study aimed to evaluate the survival and growth of 12-year forest plantations with Tabebuia rosea (rosy trumpet), and Swietenia macrophylla (Mahogany tree), established in four localities in the states of Colima, Nayarit, and Jalisco. Statistical differences in survival, normal diameter, height, volume, and their annual increments were assessed among species and localities through analysis of variance and Tukey-test. Results indicated that the survival of $T$. rosea ranged from 35 to $82 \%$, whereas in S. macrophylla it ranged from 79 to $83 \%$. There were significant differences among localities $(P<$ 0.05 ) in the evaluated growth variables. $T$. rosea stood out by its normal diameter, height, and volume in locality IV (Coast of Jalisco), whereas S. macrophylla had a better development in normal diameter and volume in locality II (EI Verdineño), possibly by the effect of soil type in these sites. These results suggest that sites with edafoclimatic conditions similar to these localities are appropriate for the establishment of forest plantations with both tropical species in central-western Mexico.
\end{abstract}

Key words: Mahogany tree, forest growth, tropical plantations, rosy trumpet, silviculture, survival.

\section{Resumen}

El éxito en el establecimiento y desarrollo de las plantaciones forestales depende de que las especies sean las adecuadas para las condiciones edafoclimáticas de los sitios de plantación. El objetivo de este estudio fue evaluar la supervivencia y crecimiento de plantaciones de 12 años con Tabebuia rosea (rosa morada) y Swietenia macrophylla (caoba), en cuatro localidades de Jalisco, Colima y Nayarit. Se determinó la diferencia estadística entre localidades con análisis de varianza y prueba de comparación de medias de Tukey, para las variables supervivencia, diámetro normal, altura, volumen y sus incrementos medios anuales. Los resultados indican que la supervivencia de $T$. rosea varió de 35 a $82 \%$ y en $S$. macrophylla entre 79 y $83 \%$. Hubo diferencias significativas $(p<0.05)$ entre localidades en las variables de crecimiento. $T$. rosea destacó por su diámetro normal, altura y volumen en la localidad IV (Costa de Jalisco), mientras que S. macrophylla tuvo un mejor desarrollo en diámetro normal y volumen en la localidad II (EI Verdineño); posiblemente, por el efecto del tipo de suelo en estos sitios. Los resultados sugieren que lugares bajo condiciones edafoclimáticas similares a esas localidades son aptos para el establecimiento de plantaciones forestales con ambas especies tropicales en el Centro-Occidente de México.

Palabras clave: Caoba, crecimiento forestal, plantaciones tropicales, rosa morada, silvicultura, supervivencia.

Fecha de recepción/ Reception date: 21 de septiembre de 2020

Fecha de aceptación/Acceptance date: 21 de julio de 2021

${ }^{1}$ Instituto Nacional de Investigaciones Forestales, Agrícolas y Pecuarias, CIR- Pacífico Centro, Campo Experimental Centro Altos de Jalisco. México.

2 Instituto Nacional de Investigaciones Forestales, Agrícolas y Pecuarias, CIR- Pacífico Centro, Campo Experimental Uruapan. México.

${ }^{3}$ Instituto Nacional de Investigaciones Forestales, Agrícolas y Pecuarias, CIR- Norte,Campo Experimental Saltillo. México.

${ }^{4}$ Instituto de Investigaciones en Ecosistemas y Sustentabilidad, UNAM. México.

*Autor para correspondencia; correo-e: jsaenz@cieco.unam.mx 


\section{Introducción}

Las plantaciones forestales comerciales (PFC) representan una alternativa para disminuir la presión sobre los bosques, asegurar el abastecimiento de materias primas a la industria forestal y generar servicios ambientales como la disminución de la erosión, aumento de la infiltración, captura de carbono, refugio de flora y fauna, aportación de recursos económicos y fuentes de empleo rural (Guerra y Galicia, 2017).

Para lograr el éxito en el establecimiento y desarrollo de las plantaciones forestales se requiere que las especies que se introduzcan sean adecuadas para las condiciones edafoclimáticas de la región, tengan alto rendimiento de materia prima, sean rentables y de interés para los productores o inversionistas (Arteaga y Castelán, 2008).

Además, es necesario utilizar semilla y planta de alta calidad y aplicar un manejo silvícola oportuno; decisiones que serán mejores, si se basan en resultados de plantaciones experimentales o de aquéllas evaluadas técnicamente, ya que constituyen una herramienta para mejorar las acciones de planificación para el establecimiento y manejo de las PFC (Pérez et al., 2012).

Las especies nativas de alto valor comercial y de rápido crecimiento pueden maximizar la producción de las PFC. Tabebuia rosea (Bertol.) DC. (rosa morada) y Swietenia macrophylla King. (caoba) son dos especies taxones nativos de importancia económica que se han establecido en PFC en el Centro-Occidente de México. La rosa morada se utiliza en la fabricación de muebles, artesanías, en herbolaria y como ornamental (Pineda et al., 2016); mientras que, la caoba se considera madera preciosa por su veteado; se usa como ornamental, medicinal, para obtener tanino o colorante, en instrumentos musicales, artículos torneados y artesanías. Por su durabilidad, también se le emplea en la fabricación de embarcaciones livianas (Negreros et al., 2014).

Con la finalidad de tener bases técnicas y científicas para elaborar una correcta planeación del establecimiento y manejo de nuevas PFC con estas especies, es necesario 
determinar la supervivencia y crecimiento bajo diferentes condiciones edafoclimáticas de los sitios de plantación. El objetivo del presente trabajo fue evaluar la supervivencia, crecimiento e incrementos de plantaciones forestales con $T$. rosea y S. macrophylla, a 12 años de establecidas en los estados de Colima, Nayarit y Jalisco. La hipótesis planteada es la existencia de diferencias en el crecimiento e incremento de ambos taxones, en función de las condiciones edafoclimáticas de los sitios de plantación.

\section{Materiales y Métodos \\ Área de estudio}

Las plantaciones forestales evaluadas se ubican en terrenos que forman parte del Centro de Investigación Regional Pacífico Centro (CIR-Pac) del Instituto Nacional de Investigaciones Forestales, Agrícolas y Pecuarias en cuatro localidades ubicadas en los estados de Colima, Nayarit y Jalisco (Figura 1), donde se establecieron parcelas divididas con 288 árboles de T. rosea y 288 árboles de S. macrophylla, bajo una densidad de plantación de 625 árboles ha-1, con un diseño de marco real y espaciamiento de $4 \times 4 \mathrm{~m}$, en ambas especies. Las semillas fueron recolectadas de poblaciones naturales cercanas a cada sitio. En la localidad I (Tecomán), las plantaciones recién establecidas fueron atacadas por la hormiga arriera (Atta sp.), lo cual podría haber influido en el establecimiento y crecimiento de las especies. Las condiciones edafoclimáticas de las cuatro localidades se presentan en el Cuadro 1. 


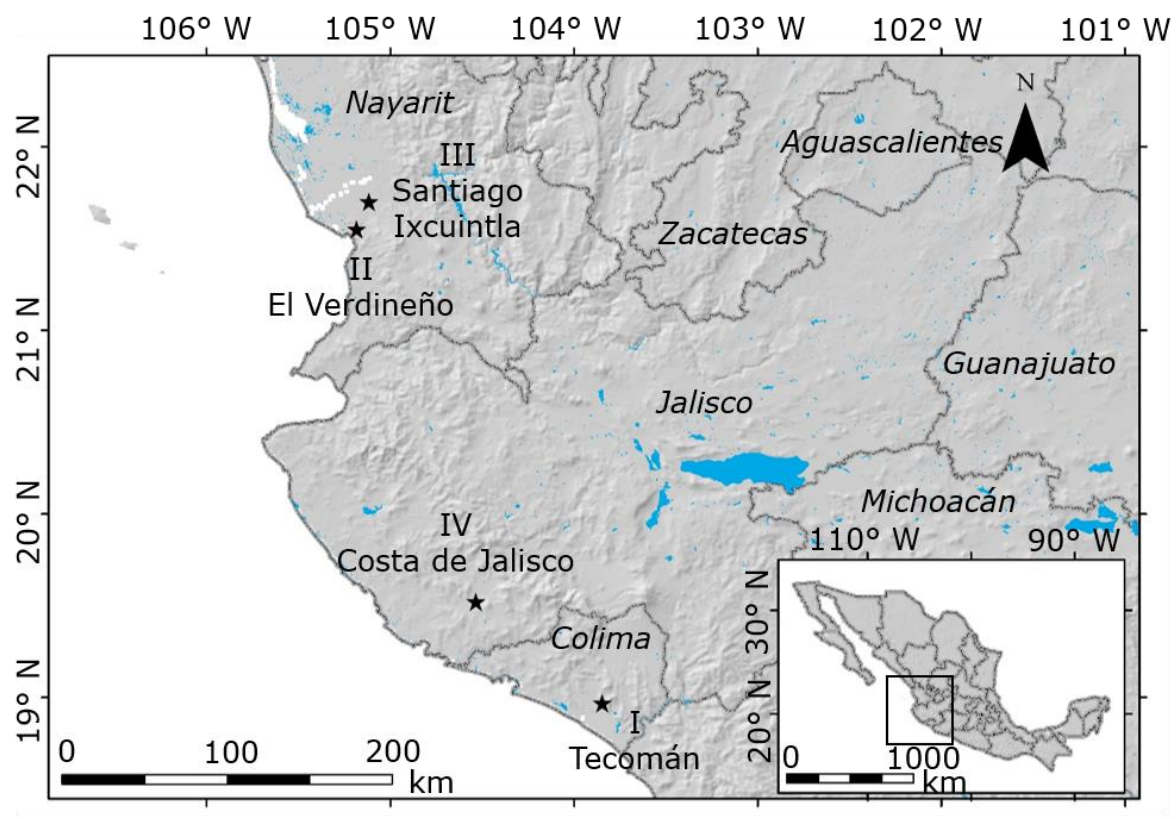

Figura 1. Localización de las plantaciones evaluadas con Tabebuia rosea (Bertol.) DC. y Swietenia macrophylla King en el Centro-Occidente de México.

Cuadro 1. Ubicación y condiciones edafoclimáticas de las localidades con plantaciones de Tabebuia rosea (Bertol.) DC. y Swietenia macrophylla King, en el Centro-Occidente de México.

\begin{tabular}{|c|c|c|c|c|c|c|}
\hline Localidad/estado & Coordenadas & $\begin{array}{c}\text { Altitud } \\
(\mathbf{m})\end{array}$ & Clima & $\begin{array}{c}\text { Temperatura media } \\
\left({ }^{\circ} \mathrm{C}\right)\end{array}$ & $\begin{array}{c}\text { Precipitación } \\
(\mathbf{m m})\end{array}$ & Tipo de suelo y textura \\
\hline $\begin{array}{c}\text { I } \\
\text { C. E. Tecomán, } \\
\text { Colima }\end{array}$ & $\begin{array}{l}18^{\circ} 55^{\prime} \mathrm{LN} \\
103^{\circ} 53^{\prime} \mathrm{LO}\end{array}$ & 40 & $\begin{array}{l}\text { cálido sub- } \\
\text { húmedo }\end{array}$ & 26 & 750 & Chernozem, franca \\
\hline $\begin{array}{l}\text { II } \\
\text { S. E. El Verdineño, } \\
\text { Nayarit }\end{array}$ & $\begin{array}{l}21^{\circ} 33^{\prime} \text { LN } \\
105^{\circ} 11^{\prime} \text { LO }\end{array}$ & 50 & $\begin{array}{l}\text { cálido sub- } \\
\text { húmedo }\end{array}$ & 24 & 1200 & Gleysol, franco-arcillosa \\
\hline $\begin{array}{c}\text { III } \\
\text { C. E. Santiago } \\
\text { Ixcuintla, Nayarit }\end{array}$ & $\begin{array}{l}21^{\circ} 42^{\prime} \text { LN } \\
105^{\circ} 07^{\prime} \text { LO }\end{array}$ & 60 & $\begin{array}{l}\text { cálido sub- } \\
\text { húmedo }\end{array}$ & 24 & 1200 & Cambisol, franco-arenosa \\
\hline $\begin{array}{c}\text { IV } \\
\text { S. E. Costa de } \\
\text { Jalisco, Jalisco }\end{array}$ & $\begin{array}{l}19^{\circ} 31^{\prime} \text { LN } \\
104^{\circ} 32^{\prime} \text { LO }\end{array}$ & 298 & $\begin{array}{l}\text { cálido sub- } \\
\text { húmedo }\end{array}$ & 25.2 & 1012 & Feozem háplico, limo-arcillosa \\
\hline
\end{tabular}

Fuente: Rueda (2008).

C. E. = Campo experimental; S. E. = Sitio experimental. 


\section{Toma de datos}

En cada una de las parcelas de las localidades del área de estudio, se hizo el inventario de los árboles sobrevivientes que se establecieron 12 años atrás. A todos los individuos se les midió la altura $(\mathrm{m})$ con clinómetro marca Suunto modelo Pm5/360pc y el diámetro normal $(\mathrm{cm})$ a la altura de $1.30 \mathrm{~m}$ con cinta diamétrica marca Jackson MS modelo Forestry Suppliers Inc; en los árboles bifurcados, el diámetro se midió por debajo de $1.30 \mathrm{~m}$ ( $2 \%$ de los árboles sobrevivientes), y se decidió considerarlos como dos ejemplares ( $\mathrm{A}$ y $\mathrm{B}$ ). El volumen individual se estimó de acuerdo a la metodología de Romahn y Ramírez (2010), en la que los árboles se subdividieron en trozas o secciones, mediante mediciones con el relascopio de Bitterlich modelo RLO00, y su volumen se calculó con la ecuación de Smalian:

$$
V=\frac{\left(S_{1}+S_{2}\right) * L}{2}
$$

Donde:

$V=$ Volumen de la troza $\left(\mathrm{m}^{3}\right)$

$S_{1}=$ Área superior de la troza $\left(\mathrm{m}^{2}\right)$

$S_{2}=$ Área inferior de la troza $\left(\mathrm{m}^{2}\right)$

$L=$ Longitud de la troza $(\mathrm{m})$

El volumen del árbol se calculó con la suma del volumen estimado de cada una de las trozas. Posteriormente, se estimó el volumen por hectárea, localidad y especie, al multiplicar el volumen promedio por la densidad del arbolado. 
Por último, se estimó el incremento medio anual del diámetro normal (IMAD), el incremento medio anual de la altura (IMAA) y el incremento medio anual del volumen ha $^{-1}$ (IMAV), dividiendo los valores de diámetro normal, altura y diámetro entre los 12 años de edad de las plantaciones.

\section{Procesamiento y análisis de información}

La normalidad de los datos se verificó mediante una prueba de Shapiro-Wilks en cada especie, para las variables diámetro normal ( $T$. rosea: $p=0.0103 ; S$. macrophylla: $p=0.0023$ ), altura ( $T$. rosea: $p=0.1594 ; S$. macrophylla: $p=0.2706$ ) y volumen $\left(T\right.$. rosea: $p=3.11 \times 10^{-5} ;$ S. macrophylla: $p=7.146 \times 10^{-7}$ ). La homogeneidad de varianzas se evaluó con una prueba de Bartlett para el diámetro normal ( $T$. rosea: $p=0.4989$; S. macrophylla: $p=0.0193$ ), altura ( $T$. rosea: $p=$ 0.5341 ; S. macrophylla: $p=0.0471)$ y volumen $\left(T\right.$. rosea: $p=7.471 \times 10^{-3} ; S$. macrophylla: $\left.p=1.725 \times 10^{-8}\right)$.

Las variables de ambos taxones fueron transformadas con logaritmo natural para su normalización: diámetro normal ( $T$. rosea: $p=0.1123$; S. macrophylla: $p=0.0551$ ), altura ( $T$. rosea: $p=0.2601 ;$ S. macrophylla: $p=0.4771$ ) y volumen ( $T$. rosea: $p=$ 0.275 ; S. macrophylla: $p=0.0497)$; se obtuvieron varianzas más homogéneas en el diámetro normal ( $T$. rosea: $p=0.1235$; S. macrophylla: $p=0.0337$ ), altura ( $T$. rosea: $p=0.5191 ;$ S. macrophylla: $p=0.7677$ ) y volumen ( $T$. rosea: $p=0.0981 ; S$. macrophylla: $p=0.437)$.

El análisis de varianza (ANOVA) y una comparación de medias con la prueba de Tukey se utilizaron para evaluar diferencias en diámetro normal, altura y volumen entre localidades para cada especie y entre las especies por localidad, con un nivel de confianza de $95 \%$; los análisis se hicieron en el lenguaje de programación R versión 
3.4.3 (R Core Team, 2017). El modelo estadístico (Norman y Steiner, 1996) se describe a continuación:

$$
Y_{i j}=\mu+A_{i}+E_{i j}
$$

Donde:

$Y_{i j}=$ Variable que representa el valor de la respuesta en la j-ésima observación del $i$ ésimo tratamiento

$\mu=$ Constante de la respuesta media de la variable $Y$

$A_{i}=$ Efectos del tratamiento $i(i=4$ localidades o 2 especies $)$

$E_{i j}=$ Error experimental

\section{Resultados}

\section{Supervivencia}

La supervivencia de $T$. rosea fue menor en la localidad I con $35 \%$ y en el resto de las localidades varió de 70 a 82 \%. En el caso de S. macrophylla, el intervalo de variación fue menor, de 79 a 83 \% de supervivencia y el mayor porcentaje se registró en la localidad I (Cuadro 2). 
Cuadro 2. Supervivencia, diámetro normal, altura, volumen por hectárea e incrementos medios anuales en plantaciones forestales con Tabebuia rosea (Bertol.) DC. y Swietenia macrophylla King en Colima, Nayarit y Jalisco, a 12 años de establecidas.

\begin{tabular}{|c|c|c|c|c|}
\hline \multirow[b]{2}{*}{ Especie } & \multicolumn{4}{|c|}{ Localidad } \\
\hline & $\begin{array}{c}\text { I } \\
\text { Tecomán } \\
\text { (Colima) }\end{array}$ & $\begin{array}{c}\text { II } \\
\begin{array}{c}\text { EI Verdineño } \\
\text { (Nayarit) }\end{array} \\
\end{array}$ & $\begin{array}{c}\text { III } \\
\begin{array}{c}\text { Santiago Ixcuintla } \\
\text { (Nayarit) }\end{array}\end{array}$ & $\begin{array}{c}\text { IV } \\
\begin{array}{c}\text { Costa de Jalisco } \\
\text { (Jalisco) }\end{array}\end{array}$ \\
\hline \multicolumn{5}{|l|}{ Supervivencia (\%) } \\
\hline $\begin{array}{l}\text { Tabebuia rosea (Bertol.) } \\
\text { DC. }\end{array}$ & 35 & 80 & 70 & 82 \\
\hline $\begin{array}{l}\text { Swietenia macrophylla } \\
\text { King }\end{array}$ & 83 & 79 & 79 & 82 \\
\hline \multicolumn{5}{|l|}{ Densidad (árboles ha-1) } \\
\hline $\begin{array}{l}\text { Tabebuia rosea (Bertol.) } \\
\text { DC. }\end{array}$ & 218 & 500 & 437 & 512 \\
\hline $\begin{array}{l}\text { Swietenia macrophylla } \\
\text { King }\end{array}$ & 518 & 493 & 493 & 512 \\
\hline \multicolumn{5}{|l|}{ Diámetro normal $(\mathrm{cm})$} \\
\hline $\begin{array}{l}\text { Tabebuia rosea (Bertol.) } \\
\text { DC. }\end{array}$ & $19.87^{a}$ & $14.17^{\mathrm{a}}$ & $17.92^{\mathrm{a}}$ & $27.96^{b}$ \\
\hline $\begin{array}{l}\text { Swietenia macrophylla } \\
\text { King }\end{array}$ & $17.04^{\mathrm{a}}$ & $21.89^{b}$ & $15.02^{\mathrm{a}}$ & $15.96^{a}$ \\
\hline \multicolumn{5}{|l|}{ IMAD (cm año $\left.{ }^{-1}\right)$} \\
\hline $\begin{array}{l}\text { Tabebuia rosea (Bertol.) } \\
\text { DC. }\end{array}$ & $1.65^{\mathrm{a}}$ & $1.18^{\mathrm{a}}$ & $1.49^{a}$ & $2.33^{b}$ \\
\hline $\begin{array}{l}\text { Swietenia macrophylla } \\
\text { King }\end{array}$ & $1.42^{\mathrm{a}}$ & $1.82^{\mathrm{b}}$ & $1.25^{\mathrm{a}}$ & $1.33^{\mathrm{a}}$ \\
\hline \multicolumn{5}{|l|}{ Altura (m) } \\
\hline $\begin{array}{l}\text { Tabebuia rosea (Bertol.) } \\
\text { DC. }\end{array}$ & $9.31^{\mathrm{a}}$ & $9.49^{a}$ & $7.16^{b}$ & $10.00^{\mathrm{a}}$ \\
\hline $\begin{array}{l}\text { Swietenia macrophylla } \\
\text { King }\end{array}$ & $7.74^{\mathrm{a}}$ & $7.48^{\mathrm{a}}$ & $8.00^{a}$ & $7.04^{b}$ \\
\hline \multicolumn{5}{|l|}{ IMAA $(m$ año-1 $)$} \\
\hline $\begin{array}{l}\text { Tabebuia rosea (Bertol.) } \\
\text { DC. }\end{array}$ & $0.77^{a}$ & $0.79^{a}$ & $0.59^{b}$ & $0.83^{a}$ \\
\hline $\begin{array}{l}\text { Swietenia macrophylla } \\
\text { King }\end{array}$ & $0.64^{\mathrm{a}}$ & $0.62^{\mathrm{a}}$ & $0.66^{\mathrm{a}}$ & $0.58^{b}$ \\
\hline \multicolumn{5}{|l|}{ Volumen $\left(\mathrm{m}^{3} \mathrm{ha}^{-1}\right)$} \\
\hline $\begin{array}{l}\text { Tabebuia rosea (Bertol.) } \\
\text { DC. }\end{array}$ & $27.41^{\mathrm{a}}$ & $35.94^{\mathrm{a}}$ & $38.39^{\mathrm{a}}$ & $128.03^{b}$ \\
\hline $\begin{array}{l}\text { Swietenia macrophylla } \\
\text { King }\end{array}$ & $49.28^{\mathrm{a}}$ & $55.92^{\mathrm{b}}$ & $34.64^{\mathrm{a}}$ & $32.27^{a}$ \\
\hline \multicolumn{5}{|l|}{ IMAV $\left(\mathrm{m}^{3} \mathrm{ha}^{-1} \mathrm{año} \mathrm{o}^{-1}\right)$} \\
\hline $\begin{array}{l}\text { Tabebuia rose (Bertol.) } \\
\text { DC.a }\end{array}$ & $2.28^{\mathrm{a}}$ & $2.99^{a}$ & $3.19^{a}$ & $10.66^{\mathrm{b}}$ \\
\hline $\begin{array}{l}\text { Swietenia macrophylla } \\
\text { King }\end{array}$ & $4.10^{\mathrm{a}}$ & $4.66^{b}$ & $2.88^{a}$ & $2.68^{\mathrm{a}}$ \\
\hline
\end{tabular}

IMAD = Incremento medio anual del diámetro normal, IMAA = Incremento medio anual de la altura, IMAV = Incremento medio anual del volumen, a = Localidad sin diferencia significativa, $b=$ Localidad con diferencia significativa, según prueba de Tukey entre localidades. 


\section{Crecimiento e incremento medio anual en diámetro normal}

En T. rosea, el diámetro normal varió de 14.17 a 27.96 cm y en S. macrophylla de 15.02 a $21.89 \mathrm{~cm}$. Se identificaron diferencias significativas entre localidades en ambas especies; T. rosea $\left(p=9.78 \times 10^{-16}\right)$, la localidad IV presentó estadísticamente el mayor diámetro normal, sitio que se distingue por situarse en la altitud más alta (298 msnm), con un suelo Feozem háplico. El diámetro normal de $S$. macrophylla fue significativamente superior ( $p=$ $2 \times 10^{-16}$ ) en la localidad II, cuyo suelo corresponde a un Gleysol.

En cada localidad hubo diferencias significativas entre ambas especies (localidad I: $p=4.84 \times 10^{-3}$; localidad II $: p=6.15 \times 10^{-14}$; localidad III: $p=3.3 \times 10^{-3}$; localidad IV: $p=2 \times 10^{-16}$ ); y $T$. rosea sobresalió, con respecto a $S$. macrophylla, excepto en la localidad II donde esta última registro el valor más alto de diámetro normal.

La variable IMAD presentó diferencias significativas entre localidades; en $T$. rosea los valores variaron entre 1.18 y $2.33 \mathrm{~cm}$ año-1, con el máximo valor en la localidad IV $\left(p=9.78 \times 10^{-16}\right)$; en S. macrophylla el intervalo del IMAD fue de 1.25 a $1.82 \mathrm{~cm}$ año-1; al respecto, destacó la localidad II $\left(p=2 \times 10^{-16}\right)$ (Cuadro 2$)$.

\section{Crecimiento e incremento medio anual en altura}

La altura de $T$. rosea varió entre 7.16 y $10.00 \mathrm{~m}$; mientas que, la de $S$. macrophylla fue de 7.04 a $8.00 \mathrm{~m}$. Entre localidades existió diferencia significativa, en las que la altura de $T$. rosea fue estadísticamente menor $(p=0.0416)$ en la localidad III, con suelo Cambisol; en la localidad IV, la altura de $S$. macrophylla fue significativamente menor $\left(p=3.64 \times 10^{-14}\right)$. 
En las localidades se detectaron diferencias significativas entre especies (localidad I: $p=8.71 \times 10^{-5}$; localidad II: $p=5.03 \times 10^{-5}$; localidad III : $p=0.0281$; localidad IV: $\left.p=2.68 \times 10^{-15}\right)$; en las localidades I, II y IV $T$. rosea fue superior; mientras que, en la localidad III destacó S. macrophylla.

Para el IMAA se obtuvieron diferencias significativas entre localidades, para $T$. rosea ( $p=0.0416)$ fluctuó entre 0.59 y $0.83 \mathrm{~m}$ año-1, con los mayores valores en la localidad IV; y en $S$. macrophylla $\left(p=3.64 \times 10^{-14}\right)$ entre 0.58 y $0.66 \mathrm{~m}^{2}$ ño-1, con el máximo IMAA en la III (Cuadro 2).

\section{Crecimiento e incremento medio anual en volumen ha ${ }^{-1}$}

El volumen ha-1 en $T$. rosea varió de 27.41 a $128.03 \mathrm{~m}^{3}$ ha-1 y en $S$. macrophylla de 32.27 a $55.92 \mathrm{~m}^{3} \mathrm{ha}^{-1}$. Diferencias significativas se registraron entre localidades en ambas especies. T. rosea tuvo mayor volumen en la IV $\left(p=1.95 \times 10^{-10}\right)$ y $S$. macrophylla en la II $\left(p=2 \times 10^{-16}\right)$.

En las localidades I, II y IV hubo diferencias significativas entre los taxones (localidad I: $p=0.0166$; localidad II $: p=5.18 \times 10^{-6}$; localidad IV: $p=2 \times 10^{-16}$ ); $T$. rosea fue dominante en las localidades I y II; mientras que, $S$. macrophylla fue mejor en la localidad IV; en la III no hubo diferencias significativas entre especies ( $p=0.283$ ).

EI IMAV presentó diferencias significativas entre localidades en los dos taxa; en $T$. rosea $\left(p=1.95 \times 10^{-10}\right)$ fue de 2.28 a $10.67 \mathrm{~m}^{3} \mathrm{ha}^{-1}$ año-1, con el mejor resultado en la localidad IV, y en S. macrophylla $\left(p=2 \times 10^{-16}\right)$ entre 2.69 a $4.66 \mathrm{~m}^{3} \mathrm{ha}^{-1}$ año1 , con el mayor valor en la II (Cuadro 2). 


\section{Discusión}

El crecimiento de las especies es el resultado de la influencia de las características edafoclimáticas de los sitios de plantación y de su genética o crecimiento natural (Martínez y Álvarez, 1995). Por ejemplo, T. rosea en etapas iniciales es de rápido crecimiento, pero presenta patrones de bifurcación muy variables que pueden afectar el crecimiento en altura y en diámetro normal (Borchert y Tomlinson, 1984).

Los porcentajes de supervivencia de las plantaciones de este estudio se consideraron altos en ambas especies, excepto para $T$. rosea en la localidad I. La supervivencia de $S$. macrophylla en la región de estudio fue inferior al de plantaciones de 13 años en Tabasco (100 \%), donde prevalece mayor precipitación media anual, de hasta 2290 mm anuales y los suelos presentan mayor contenido de humedad (Fluvisol éutrico, Cambisol y Gleysol mólico) (Pérez et al., 2012).

El diámetro normal de S. macrophylla fue mayor al registrado en plantaciones del sur de Jalisco $(10.2 \mathrm{~cm}$ ) (Rueda et al., 2014); mientras que, $T$. rosea fue similar al de plantaciones de 14 años de la misma región (15 a $24 \mathrm{~cm}$ ) (Rueda et al., 2010); pero su IMAD resultó superior, en comparación al de árboles de esta especie en el estado de Guerrero (0.58 cm año-1), sujetos a condiciones más secas (Pineda et al., 2016). Sin embargo, el diámetro normal de los dos taxones estudiados fue inferior al de individuos de 11 y 13 años de Tectona grandis L. f. en Nayarit (25 a $28 \mathrm{~cm}$ ), en sitios con suelos lacustres de alto contenido de humedad (Luvisol y Solonchak) (Vincent, 2018).

La altura que alcanzó $T$. rosea en las localidades I, II y IV fue menor a la registrada en una plantación de 14 años ubicada en la Costa de Jalisco (12.8 m) (Distancia et al., 2008); y superó la de Cedrela odorata L. (8.19 m) en plantaciones de Tecomán (Orozco et al., 2010), pero fue inferior a la registrada en plantaciones de 11 y 13 años de $T$. grandis en Nayarit (15 y 25 m, respectivamente) (Vincent, 2018). La altura de S. macrophylla también fue menor a la citada para plantaciones de 11 a 16 años 
en Tabasco (14-23 m), donde la precipitación media anual es mayor, lo cual promueve el desarrollo de T. grandis (Pérez et al., 2012).

El volumen de los árboles de $T$. rosea en la localidad IV superó al de plantaciones de 11 a 13 años de $T$. grandis (Vincent, 2018) y el IMAV al de plantaciones de Gmelina arborea Roxb. ex. Sm. (10.5 $\left.\mathrm{m}^{3} \mathrm{ha}^{-1} \mathrm{año}^{-1}\right)$ en sitios con suelos pobres del estado de Tabasco (Martínez et al., 2015). En el caso de S. macrophylla, el volumen de los árboles fue similar al estimado en una plantación de 11 a 13 años en Tabasco (37-98 $\mathrm{m}^{3} \mathrm{ha}^{-1}$ ) (Pérez et al., 2012).

Los resultados de este estudio indican que $T$. rosea fue mejor en la localidad IV (diámetro normal, altura y volumen) y S. macrophylla en la II (diámetro normal y volumen) y IV (altura); lo cual sugiere que las especies pueden tener mejor rendimiento en función de las condiciones edafoclimáticas de cada sitio (Méndez y Vanegas, 2016). La principal variable distinta entre las localidades fue el tipo de suelo, pues los tipos Cambisol (localidad II) y Feozem (localidad IV) habrían promovido un mejor desarrollo de las especies evaluadas.

Los cambisoles, generalmente, destacan por su alto contenido en nutrientes como hierro, carbonato de calcio y manganeso; mientras que, los feozem son ricos en materia orgánica, calcio y potasio (Vivanco et al., 2010). Es importante evaluar en estudios posteriores, el efecto del tipo de suelo y la precipitación sobre el desarrollo de estas especies tropicales. En el segundo caso, habría indicios de que la supervivencia de $S$. macrophylla sería mayor en lugares con mayor precipitación media anual, como en Tabasco (Pérez et al., 2012); y diámetro normal menor en $T$. rosea en sitios más secos, como en Guerrero (Pineda et al., 2016).

Lo anterior confirma que el rendimiento de las especies tropicales está sujeto a condiciones edafoclimáticas muy específicas. Por ejemplo, C. odorata en la Costa de Jalisco se desarrolla bien en altitudes cercanas a 300 msnm; clima cálido subhúmedo, con precipitación media anual de 1100 mm; y suelo Feozem háplico (Rueda et al., 2014); mientras que G. arborea en el trópico seco del estado de Michoacán, 
en altitudes de $500 \mathrm{~m}$, clima cálido subhúmedo, suelo Vertisol y pendientes suaves (Muñoz et al., 2009).

Los resultados de este estudio permitieron identificar que $T$. rosea y S. macrophylla tienen amplio potencial para establecerse en PFC dentro del Centro-Occidente de México; la primera especie en la Costa de Jalisco (región de La Huerta) y la segunda en la Costa de Nayarit (región de El Verdineño). Por tanto, la evaluación de plantaciones forestales constituye una herramienta fiable para determinar las zonas donde se deben establecer. Sin embargo, es indispensable evaluar qué sitios cumplen con las características edafoclimáticas para otras especies forestales de importancia comercial y para la restauración ecológica.

\section{Conclusiones}

La evaluación de variables como la supervivencia, diámetro normal, IMAD, altura, IMAA, volumen e IMAV en plantaciones experimentales de $T$. rosea y S. macrophylla en el Centro-Occidente de México permitió identificar que las mejores localidades para establecer PFC son la Costa de Jalisco (localidad IV), en el caso de la primera especie; y la Costa de Nayarit en el caso de la segunda (localidad II).

El tipo de suelo podría ser el factor determinante sobre las diferencias en las variables entre localidades, por lo que es importante conocer su efecto sobre el desarrollo de ambos taxones tropicales en estudios posteriores. Además, se confirmó que las especies tienen diferente desempeño de acuerdo a las condiciones edafoclimáticas particulares de los sitios de plantación, por lo que es importante ponderar el crecimiento y desarrollo de las especies en condiciones ambientales específicas, previo a su establecimiento en PFC masivas o en plantaciones de restauración. 


\section{Agradecimientos}

Los autores agradecen al personal de los campos experimentales de Tecomán y Santiago Ixcuintla y los sitios experimentales El Verdineño y Costa de Jalisco del Instituto Nacional de Investigaciones Forestales, Agrícolas y Pecuarias (INIFAP), y al Posgrado en Ciencias Biológicas de la Universidad Nacional Autónoma de México.

\section{Conflicto de intereses}

Los autores manifiestan no tener conflicto de intereses.

\section{Contribución por autor}

Agustín Rueda Sánchez: elaboración del proyecto y muestreo en campo, redacción del manuscrito; Juan de Dios Benavides Solorio: elaboración del proyecto y muestreo en campo; J. Trinidad Sáenz Reyes: análisis estadístico, redacción y revisión del manuscrito; H. Jesús Muñoz Flores: redacción y revisión del manuscrito; David Castillo Quiroz: redacción y revisión del manuscrito; Jesús Eduardo Sáenz Ceja: análisis estadístico y redacción del manuscrito.

\section{Referencias}

Arteaga M., B. y M. Castelán L. 2008. Evaluación dasométrica temprana de una plantación agroforestal de tres especies introducidas en el municipio de Huehuetla, Hidalgo. Revista Chapingo Serie Ciencias Forestales y del Ambiente 14(2): 105-111. https://revistas. chapingo. $\mathrm{mx} /$ forestales/?section $=$ articles\&subsec $=i$ issues $\&$ numero $=$ $38 \&$ articulo=497 ( 6 de agosto de 2020). 
Borchert, R. and P. B. Tomlinson. 1984. Architecture and crown geometry in Tabebuia rosea (Bignoniaceae). American Journal of Botany 71(7):958-969. Doi: 10.1002/j.15372197.1984.tb14162.x.

Distancia C., O., J. D. Benavides S., A. Rueda S. y A. Gallegos R. 2008. Evaluación del crecimiento de una plantación experimental de 1992 al 2005 de Cedrela odorata, Swietenia macrophylla, Enterolobium cyclocarpum y Tabebuia rosea en la costa de Jalisco. In: Carbajal S. (ed.) Avances en la Investigación Científica 2008. Centro Universitario de Ciencias Biológicas y AgropecuariasUniversidad de Guadalajara. Guadalajara, Jal. (pp. 93-102).

http://www.cucba.udg.mx/sites/default/files/publicaciones1/avances/avances2008/Agronomia/Prod uccionForestal(pp87-122)/DistanciaCarbajalOlivia/93-102.pdf (6 de diciembre de 2020).

Guerra C., V. and L. Galicia. 2017. Tropical and highland temperate forest plantations in Mexico: Pathways for climate change mitigation and ecosystem services delivery. Forests 8: 489. Doi: 10.3390/f8120489.

Martínez R., M. y E. Álvarez B. 1995. Ecología de poblaciones de plantas en una selva húmeda de México. Boletín de la Sociedad Botánica de México 56: 121-153. Doi: $10.17129 /$ botsci. 1469.

Martínez Z., P., M. Domínguez D., A. Juárez G., L. M. López L., V. de la Cruz A. y J. Álvarez J. 2015. Índice de sitio y producción maderable en plantaciones forestales de Gmelina arborea en Tabasco, México. Revista Fitotecnia Mexicana 38(4): 415425. http://www.scielo.org.mx/pdf/rfm/v38n4/v38n4a10.pdf (6 de mayo de 2020).

Méndez P., B. A. y E. A. Vanegas C. 2016. Factores de sitio y crecimiento de plantaciones de palo blanco (Tabebuia donnel-smithii Rose) en Guatemala. Revista Cubana de Ciencias Forestales 4(2): 175-185. http://cfores.upr.edu.cu/index.php/cfores/article/view/155 (3 de mayo de 2020). 
Muñoz F., H. J., V. M. Coria A., J. J. García S. y M. Balam C. 2009. Evaluación de una plantación de tres especies tropicales de rápido crecimiento en Nuevo Urecho, Michoacán. Ciencia Forestal en México 34(106): 61-87.

http://cienciasforestales.inifap.gob.mx/editorial/index.php/forestales/article/view/684 (2 de junio de 2020).

Negreros C., P., L. Cámara C., M. S. Devail, M. A. Fajvan, M. A. Mendoza B. y C. W. Mize, A. Navarro M. 2014. Silvicultura de las selvas de caoba en Quintana Roo: Criterios y recomendaciones. Comisión Nacional Forestal. México, D. F., México. 186 p. http://www.conafor.gob.mx:8080/documentos/docs/50/6577Silvicultura $\% 20$ de $\% 20$ las\%20selvas\%20de\%20caoba.pdf (29 de septiembre de 2020).

Norman, G. R. y D. Streiner. 1996. Bioestadística. Mosbi/Doyma Libros. Madrid, España. 260 p.

Orozco G., G. G., H. J. Muñoz F., J. D. Benavides S., E. A. Rubio C., A. A. Chávez D. y J. Xelhuantzi C. 2010. Evaluación de plantaciones forestales comerciales tropicales en el Estado de Colima. Folleto Técnico Núm. 22 SAGARPA. INIFAP. CIRPAC. Campo Experimental Uruapan. Uruapan, Mich., México. $52 \mathrm{p}$.

Pérez G., G., M. Domínguez D., P. Martínez Z. y J. D. Etchevers B. 2012. Caracterización dasométrica e índice de sitio en plantaciones de caoba en Tabasco, México. Madera y Bosques 18(1): 7-24. Doi: 10.21829/myb.2012.181511.

Pineda H., E., J. I. Valdez H. y C. P. Pérez O. 2016. Crecimiento en diámetro y fenología de Tabebuia rosea (Bertol.) DC. en Costa Grande, Guerrero, México. Acta Universitaria 26(4): 19-28. Doi: 10.15174/au.2016.914.

R Core Team. 2017. R project 4.3.4. https://www.r-project.org/ (16 de febrero de 2017).Romahn V., C. F. y H. Ramírez M. 2010. Dendrometría. Universidad Autónoma Chapingo. Texcoco, Mex. 294 p.

http://dicifo.chapingo.mx/pdf/publicaciones/dendrometria.pdf (6 de diciembre de 2020). 
Rueda S., A. 2008. Plantaciones forestales de especies tropicales en Jalisco, Nayarit y Colima: perspectiva productiva, ambiental y económica. Tesis de doctorado. Centro Universitario de Ciencias Biológicas y Agropecuarias, Universidad de Guadalajara. Zapopan, Jal., México. 214 p. http://repositorio.cucba.udg.mx:8080/xmlui/handle/123456789/4522 (18 de junio de 2021).

Rueda S., A., A. Gallegos R., D. González E., J. D. Benavides S., J. A. Ruiz C. y E. López A. 2010. Coeficiente de aserrío de madera en rollo de dos especies tropicales producto de plantaciones forestales. Scientia CUCBA 12: 1-10.

http://www.cucba.udg.mx/sites/default/files/publicaciones1/page_scientia_cucba/sc ientia_vol12.pdf (6 de diciembre de 2020).

Rueda S., A., A. Gallegos R., D. González E., J. A. Ruiz C., J. D. Benavides S., E. López A. y M. Acosta M. 2014. Estimación de biomasa aérea en plantaciones de Cedrela odorata L. y Swietenia macrophylla King. Revista Mexicana de Ciencias Forestales 5(25): 8-17. Doi: 10.29298/rmcf.v5i25.300.

Vincent, V. 2018. Estimación de biomasa y carbono en plantaciones de teca (Tectona grandis L. F.) por método indirecto y mapeo mediante sistemas de información geográfica. Tesis de maestría. Centro Universitario de Ciencias Exactas e Ingenierías. Universidad de Guadalajara. Guadalajara, Jal., México. 80 p.

https://mx.boell.org/sites/default/files/uploads/2018/01/tesis_de_valdimir_vincent_maestria _en_ciencia_de_productos_forestales.pdf (8 de abril de 2020).

Vivanco, J. C., J. I. Bojórquez, R. M. Murray, O. Nájera, A. Hemández y F. Flores. 2010.

Características de los principales suelos de la cuenca del río Mololoa, Tepic, Nayarit, México. Cultivos Tropicales 31(1): 32-40. http://scielo.sld.cu/pdf/ctr/v31n1/ctr05110.pdf (8 de diciembre de 2020).

\section{(c) (i) (9)}

Todos los textos publicados por la Revista Mexicana de Ciencias Forestales -sin excepción- se distribuyen amparados bajo la licencia Creative Commons 4.0 Atribución-No Comercial (CC BY-NC 4.0 Internacional), que permite a terceros utilizar lo publicado siempre que mencionen la autoría del trabajo y a la primera publicación en esta revista. 